\title{
A Novel Methodology of AC Signal Detection Using Wavelet
}

\author{
Yingli Shu ${ }^{1, a}$ \\ ${ }^{1}$ Changchun Institute Of Technology, Chuangchun, Jilin, China, 130021 \\ a shuyingli111@126.com
}

Keywords: Power System; Wavelet Transform; Neural Network; Novel Approach

\begin{abstract}
In this paper, we propose a novel AC signal detection approach to DC power system. Combining with state-of-the-art detection circuit and WNN(wavelet based neural network). Aiming at handling with the travelling wave and fault signal cannot be detected accurately with the uncertainty of signal velocity when AC signal injected. In which, the injected AC signal in DC power system is detected via the current transformers and the voltage transformers distributed in different current loops. The acquire signal acts as the original fault signal and the sub-band energy function of wavelet packet decomposition is used as secondary characteristic and the minimum distance is used as the criterion in WNN method. Experimental analysis is conducted to show the effectiveness an robustness of our approach. The final conclusion shows that detecting AC signals in DC power system is possible with our propose method.
\end{abstract}

\section{Introduction}

In power system and substations of large enterprises and plants, the DC power system is essential to the operation, monitoring and protection of substations. The normal operation of electrical equipment in substation or power grid would be affected once a severe fault occurred like AC signal injection, DC grounding etc. [1]. Of all these power failures, the AC signal mixed faults own the largest proportion, which result in the outage of a whole plant and generator tripping out [2]. Thus, relevant technical measures are developed in order to prevent the AC signals inject into DC power system and its insulation system, is of a great significance for the safety and stability of DC power system. Currently, the fault detection in DC power system mainly focused on DC power supply like uninterruptible power supply system (UPS) and high voltage direct current (HVDC) system. To the former, there are three different practical implementations for fault detection: (1) AC signal injection is used for an ungrounded battery system, and uses an AC signal source to impose an AC voltage to ground on the ungrounded battery string. (2) The balancing bridge method. (3) The unbalanced bridge method is used for grounding fault detection in positive and negative electrodes of DC power system, the faults can be detected when the voltage drops of isolation resistances is equal [3], but also has the same shortage with the method introduced above. This paper presents the AC signal detection method and a novel acquisition circuit in DC power system, firstly. And then gives an online fault detection approach with WNN applied on the bases of a theoretic analysis of regular isolation detection method and wavelet transformation combines with regular analog signal acquisition method. Finally, simulations and experiments are used to verify the correctness of the proposed method.

\section{Our Proposed Methodology}

Signal Detection Structure. There are three patterns of AC signal injects into DC power system, the first way is AC power source injects between DC positive electrode and ground, the second way is AC power source injects between DC negative electrode and ground and the last way is AC power source injects between the positive bus and negative bus. In this paper, we use AC source with maximum amplitude 220V (RMS) as test signal, the signal detection structure can be seen in Figure 1 . The Figure 1 also presents two patterns for AC signal acquisition, the first pattern uses R1, C1, R2, IL1, IL2 and VL2 as part of AC signal acquisition circuit. Once an AC signal mixed in either positive 
or negative electrode of DC power system, it would make up a circle circuit with R1, C1, R2, C2 and R3. If the output of IL1 equals to IL2 and the voltage drop in R3 is zero, we can point out that the AC signal is mixed between positive electrode and ground. In the second pattern, R4, C3, R5, C4, R6, IL3, IL4, VL3 and VL4 are used to make up another circle circuit. The location of AC signal mixed can be decided between negative electrode and ground or between positive bus and negative bus by making an analysis in voltage drops of R4 and R5. Where $\mathrm{IL}^{*}$ and $\mathrm{VL}^{*}$ respectively stand for the current sensor and the voltage sensor.
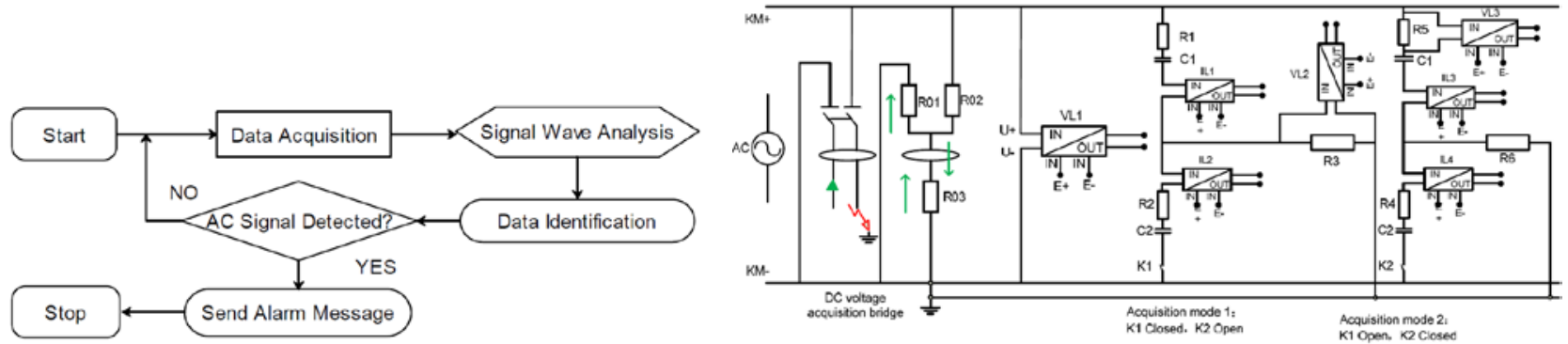

Figure.1 The Flow Diagram and Schematic Diagram

Wavelet Theory Analysis. Wavelet function is constructed through a series of basic transformation with a mother wavelet function. Let $\varphi(t)$ be a square integrable function, that is $\varphi(t) \in L^{2}(R)$. If its Fourier transform $\Psi(w)$ can satisfy the following compatibility condition:

$$
\int_{R} \frac{|\Psi(w)|^{2}}{w} d w<\infty
$$

Wavelet transform calculates the inner product between the signal $\mathrm{x}(\mathrm{t})$ with mother wavelet function and equivalent expression in time domain is given as :

$$
\begin{gathered}
f_{x}(a, \tau)=\frac{1}{\sqrt{a}} \int_{-\infty}^{\infty} x(t) \varphi^{*}\left(\frac{t-\tau}{a}\right) d t \\
f_{x}(a, \mathrm{~b})=\frac{\sqrt{a}}{2 \pi} \int_{-\infty}^{\infty} X(w) \Psi^{*}(a w) e^{j w t} d w
\end{gathered}
$$

Wavelet Neural Network. The WNN is a variety of two techniques and inherits the advantages of the neural network and wavelet transformation. The WNN topology is based on BP network; the transfer function of hidden layer nodes is the mother wavelet function; and the network signal is prior to transmission while error is back-propagation in the training process. The network topology is shown in Figure 2. In Figure $1, x_{1}, x_{2}, x_{3}, \ldots, x_{n}$ is the input vector; $y_{1}, y_{2}, y_{3}, \ldots, y_{l}$ is the predicted output; $w_{i j}$ and $w_{k j}$ are the weights connecting every layer; and $h_{j}$ is mother wavelet function [4].

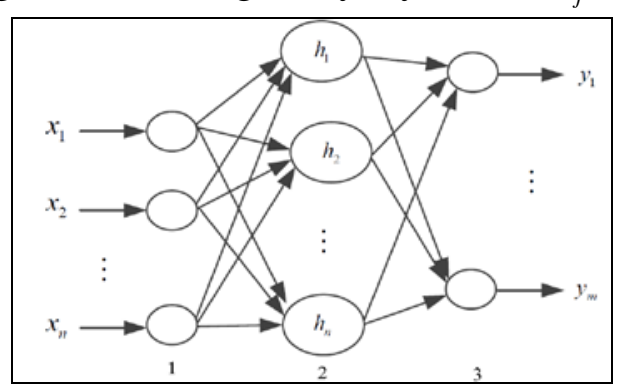

Figure.2 The Network Topology

For the input signal sequence $x=\left(\mathrm{x}_{1}, \mathrm{x}_{2}, 3, \ldots, \mathrm{x}_{n}\right)$, the output of the hidden layer is calculated as:

$$
h(j)=h_{j}\left[\frac{\sum_{i=1}^{n} w_{i j} x_{i}-b_{j}}{a_{j}}\right], \quad j=1,2, \ldots, m
$$


Where $h(j)$ is output value for the node $\mathrm{j}$ in the hidden layer; $h_{j}$ is the mother wavelet function; $w_{i j}$ is weight connecting the input layer and hidden layer; $b_{j}$ is the shift factor, and $a_{j}$ is the stretch factor for $h_{j}$. The output of the output layer is calculated as:

$$
y(k)=\sum_{i=1}^{m} w_{i k} h(i)
$$

Fault Detection with WNN. To the AC signal with a frequency of $50 \mathrm{~Hz}$, once injected into DC power system, the voltage and current can be detected as the AC signal contains harmonic with different orders. As is well known, the WNN is of significant effect in extraction of singular component. In this paper, we use the fundamental component $i_{0}$ of the injected AC signal current as the original signal for fault recognition. The wavelet coefficients of sub bands is obtained by wavelet packet transform, and uses the function of the wavelet coefficient quadratic sum as the quadratic characteristic $\mathrm{E}$ of fault pattern recognition.

$$
E=f\left(\left|d_{m}^{s, n}\right|^{2}\right)=k\left|d_{l}^{j, n}\left(i_{0}\right)\right|^{2}
$$

This paper uses the loose WNN model as training Model as is shown in Figure 3. In which, back propagation (BP) network is selected for neural network, the excitation function of hidden layer uses tangent Sigmoid function, the excitation function of output layer uses a linear function and the network training function is adaptive learning rate algorithm. The network uses the wavelet energy function $\mathrm{E}$ of the bottom sub-band energy as input after a wavelet decomposition of the original fault signal.

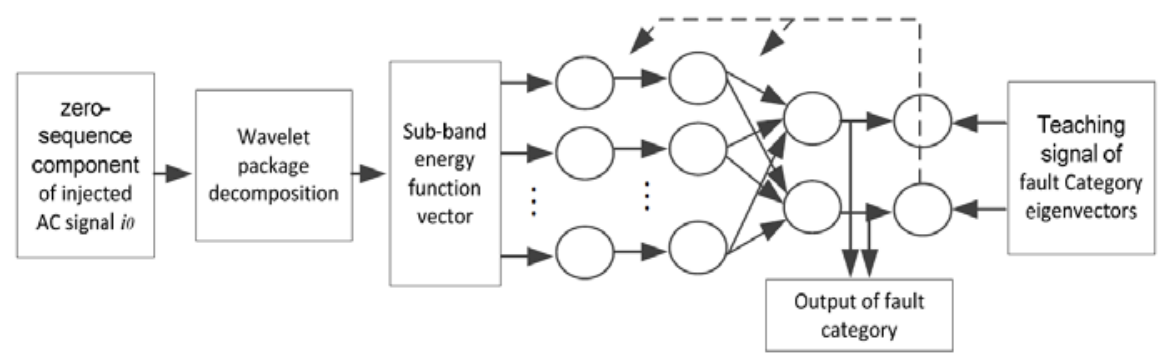

Figure.3 Structure of WNN for Fault Identification

\section{Experimental Result of the Data}

As is mentioned above, the training sample $\mathrm{X}$ is difference between the fundamental component of AC signal injected into DC circuit and the fundamental component of the parasitic AC signal inherented in DC circuit. Figure 4 shows the analyzed signal which is originally collected by using the fault detection circuit proposed in Figure 2 with between DC positive electrode and ground. The red line stands for the training sample $\mathrm{X}$, the blue line shows the fundamental component of the parasitic AC signal inherented in DC circuit and the green line stands for the fundamental component of AC signal injected into DC circuit. In this paper, we use 20001 sample data for the 3 layer wavelet deposition, and the decomposition results is shown in Figure 5:

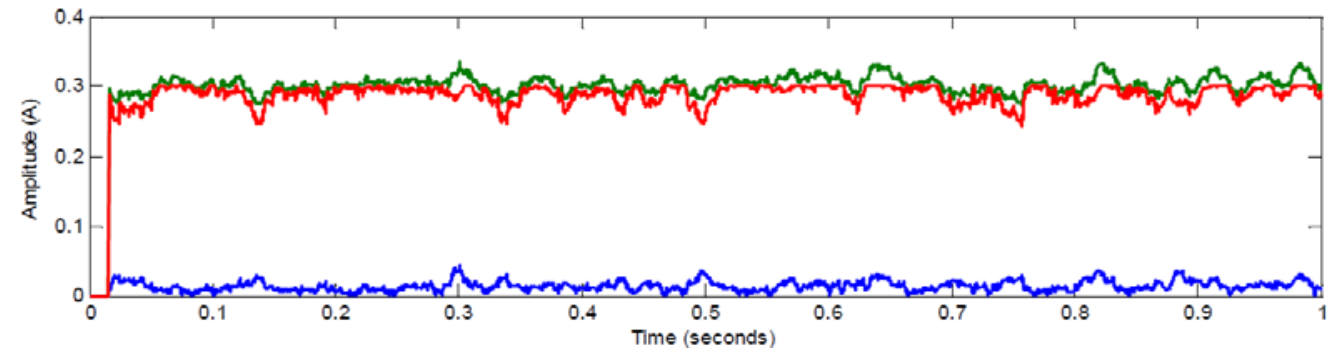

Figure.4 Fundamental Component of AC Signal 


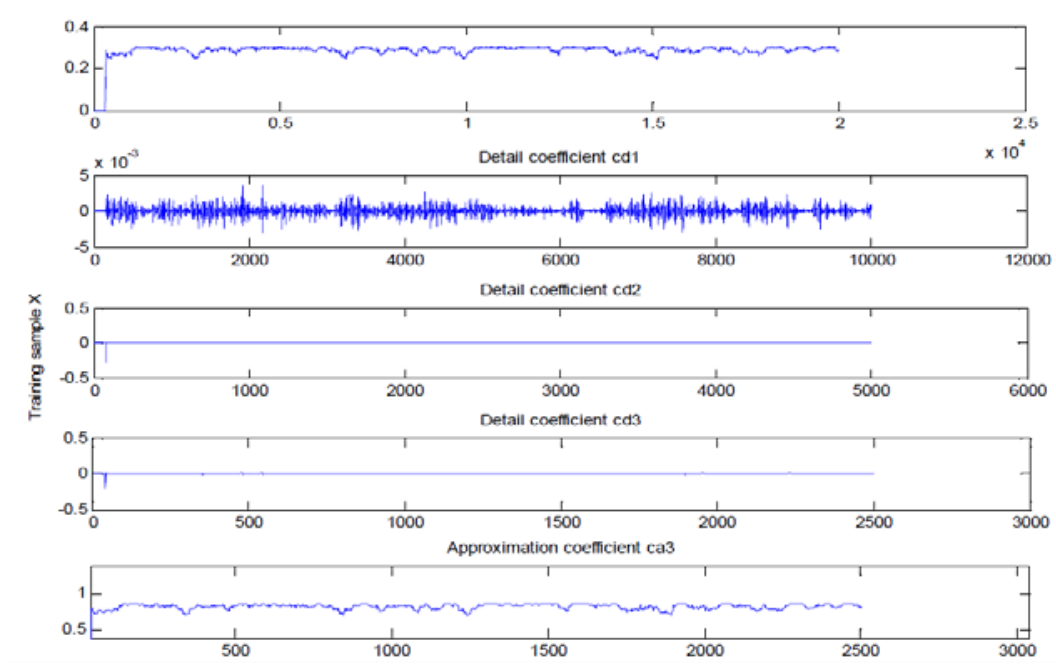

Figure.5 The 3 Layer Wavelet Deposition Results

To verify the correctness of WNN method, an AC signal injection test is conducted. Figure 6(a) is the test circuit simulation result conducted in MATLAB/Simulink, where the AC signal is injected between positive bus and ground.

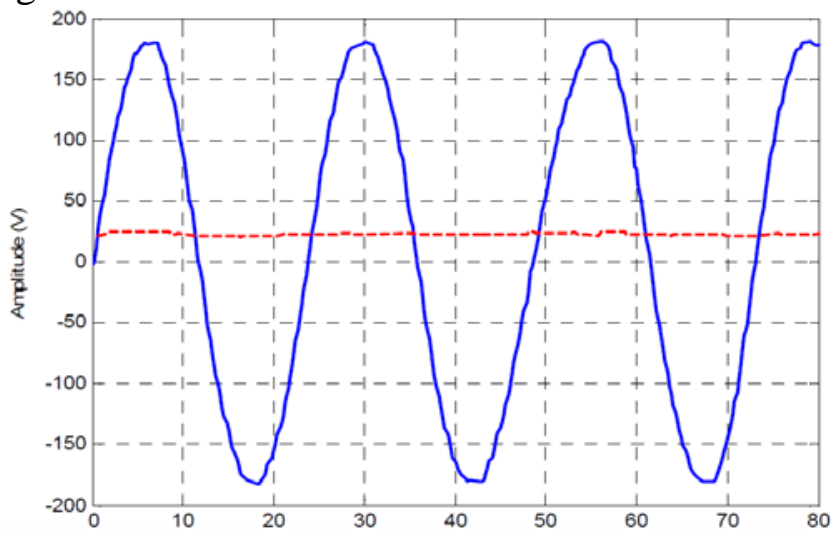

Figure.6 Simulation Wave with 100V (RMS) AC Signal Injected

\section{Summary and Conclusion}

This paper uses WNN as an effective approach to detect AC signals in DC power system, in which, the sub-band energy function of wavelet decomposition is used as eigenvector. The fault signal is accurately detected, classified and fully re-demonstrated with the premise of no additional hardware, and also realized synchronization and accuracy of AC signal detection, which is of great importance to the development of fault detection technology in DC power system.

\section{References}

[1] A Lizkano, J Ruiz. Evaluation of A New Proposal for Arcing Fault Detection Method Based on Wavelet Packet Analysis. Power Engineering Society Summer Meeting, Vancouver, Canada. 2011;

[2] YANG Xiu, CHEN Chen. Impact of HVDC Faults on Torsional Oscillation of Tuebine Generator's Shafting. Power System Technology. 2004; 28(22): 6-10.

[3] Jae-Do Park, Jared Candelaria. Fault Detection and Isolation in Low-Voltage DC-Bus Microgrid System. IEEE Transactions on Power Delivery. 2013: 28(2): 779-787.

[4] Randy K Young. Wavelet Theory and Its Applications. Springer. 1993: 222-223. 